\title{
Hybrid Group Acceptance Sampling Plan on Truncated Life Tests based on Sushila Distribution
}

\author{
Sandeep Kumar* \\ Department of Statistics, Hindu College, University of Delhi, Delhi-110007, India \\ *Corresponding author
}

\section{A B S T R A C T}

\section{Keywords \\ Consumer's risk, \\ Operating characteristics \\ function, Hybrid group, \\ Acceptance Sampling \\ Plan (HGASP), Sushila \\ distribution, Truncated \\ life test \\ Article Info \\ Accepted: \\ 04 July 2019 \\ Available Online: \\ 10 August 2019}

In this study, a hybrid group acceptance sampling plan is proposed for a truncated life test when the lifetime of a system follows Sushila distribution. The minimum group size and operating characteristics values are determined when we fixed the sample group size and testing time $\left(\mathrm{t}_{\mathrm{o}}\right)$. The operating characteristics values according to various consumers' risk requirement are presented. The results are explained with the tables and suitable examples.

\section{Introduction}

Acceptance sampling plan is an important part of statistical quality control, where we have to take a decision to accept or reject a $\log$ based on random sample selection.

If a lot is accepted we sent it to further process otherwise rejected lot may be require rework or submit with scrapped. In life testing theory, we assume quality characteristic of any product treated as random variable then it will follow a distribution. The life time of a distribution involves average life, cost, manpower, etc. are the important factors to measure of a product.
Generally, we are interested to determine the average life and minimum sample size of the product. The truncated life test part of life testing theory where to reduce cost and time, we terminate the process at a targeted time $(\mathrm{t})$ when failure items exceed over acceptance number (c). In life testing, we accept the lot if the average life of product i.e. $(\mu)$ is greater or equal to the specified life $\left(\mu_{0}\right)$, otherwise lot is rejected. Finally, life of any product follows a distribution and the behavior and characteristics of the product represent by probability density function (p.d.f.) and cumulative density function (c.d.f.) of the distribution. If we test a practice with a multiple number of items simultaneously at a 
time, these items are called group and this sampling plan is known as group acceptance sampling plan. A group acceptance sampling plan can save testing time and cost involved. Further, to determine the minimum number of items for a specified number of groups is called as hybrid group acceptance sampling plan. The HGASP is more useful than an ordinary GASP. The combination of HGASP with truncated life time distribution is known as HGASP for truncated life test.

Espstein (1954), Sobel and Tischendrof (1959), and Goode and Kao (1961) were first to discussed acceptance sampling plan based on truncated life test. Later Gupta (1960, 1962) also proposed the work with gamma and log-normal distribution Srinivasa Rao (2010) proposed the group acceptance sampling plan with lomax distribution.

Srinavasa Rao, G. (2011) proposed an extension work known as hybrid group acceptance sampling plan with log-logistic distribution. Kantam et al., (2014) developed a hybrid group acceptance sampling plan for size biased lomax distribution. Rajgopal, S. and Vijayadevi, K. (2018) proposed a hybrid group acceptance sampling plan with transmuted rayleigh distribution.

In, this paper, we have been developed a truncated acceptance sampling plan to find minimum group size and operating characteristic values with protection of producer's and consumer's risks. We assume the life time of the product follows Sushila distribution.

\section{Sushila distribution}

Shanker et al., (2013) developed a two parameter $(\mu a n d \sigma)$ continuous distribution which is known as Sushila Distribution. The probability density function (p.d.f.) of this distribution is given as:- $f(t, \mu, \sigma)=\frac{\sigma^{2}}{\mu(\sigma+1)}\left(1+\frac{t}{\mu}\right) e^{\frac{-t}{\mu} t}$,

$t>0, \mu>0, \sigma>0$.

And the cumulative distribution function (c.d.f.) of the Sushila Distribution random variable is given by:-

$F(t, \mu, \sigma)=1-\frac{\mu(\sigma+1)+\sigma t}{\mu(\sigma+1)} e^{-\frac{\sigma}{\mu} t}$

$t>0, \mu>0, \sigma>0$.

With

mean $=\frac{\mu+2}{\mu+1}\left(\frac{\mu}{\sigma}\right)$

v ariance $=\left(\frac{\mu}{\sigma}\right)^{2}\left[2\left(\frac{\mu+3}{\mu+1}\right)-\left(\frac{\mu+2}{\mu+1}\right)^{2}\right]$

Coefficient of Variation

$(\mathrm{C} . \mathrm{V})=.\sqrt{\frac{\sigma^{2}+4 \sigma+2}{\sigma+2}}$ is free from parameter

Where $\mu$ is scale and $\sigma$ is shape parameter of SD.

The purpose of this paper to obtain a hybrid group acceptance sampling plan (HGASP) based on truncated life tests when the lifetime of a product follows the two parameter SD $(\mu$, $\sigma)$ continuous distribution.

\section{Materials and Methods}

\section{Design of the proposed plan}

Let $\mu$ represent the true mean life of a product and $\mu_{0}$ denote the specified average life of the product, with consideration that the lifetime of an item follows Sushila distribution. 
A product is accepted as good if it supports the hypothesis $\mathrm{H}_{0} \mu>, \mu_{0}$ against $\mathrm{H}_{1}: \mu<\mu_{0}$. In acceptance sampling plan, the hypothesis is tested based on the number of failures from a lot as a sample in a pre-fixed time. If the number of failures more than the acceptance number we reject the lot. So, we may conclude that accept the lot with $\mu>\mu_{0}$ at fixed consumer's risk. Otherwise, we reject the lot.

Now, we propose the following acceptance sampling plan based on truncated life test:-

Select the number of testers, $r$ and assign the $r$ observation to each predefined groups, $\mathrm{g}$ and the required sample

$\mathrm{n}=\mathrm{rg}$

Acceptance number $\mathrm{c}$ is prefixed for each group and experimental time to.

Now accept the lot if number of failures is less than equal to $\mathrm{c}$ for each group.

Reject the lot if number of failures is more than $\mathrm{c}$ in any group and terminate the experiment.

Now, we are interested to determine the number of groups for various values of acceptance number $\mathrm{c}$, while termination time is assumed to be specified as $t_{0}$. The terminating time is assumed as to $=\mathrm{a} \mu_{0}$ where, $\mathrm{a}$ is constant termination ratio.

To maintain the quality of a product, we have to satisfy both producer and consumer. So it is desirable to protection of both the parties. The probability of rejecting a good lot is denoted by $\alpha$ and called producer's risk. The probability of accepting a bad lot is denoted by $\beta$ and called consumer's risk. We assumed consumer's risk $\beta$ is fixed to determine the number of testers (r) for acceptance sampling plan.
$\mathrm{P}_{\mathrm{a}}\left(\mathrm{p}_{1}\right)=\mathrm{P}\left(\mathrm{X}<\mathrm{c} \mid \mathrm{n}, \mathrm{p}_{1}\right)=1-\alpha$

$\mathrm{P}_{\mathrm{a}}\left(\mathrm{p}_{2}\right)=\mathrm{P}\left(\mathrm{X}<\mathrm{c} \mid \mathrm{n}, \mathrm{p}_{2}\right)=\beta$

Where $\mathrm{p}_{1}$ and $\mathrm{p}_{2}$ are the probabilities of failure of the producer's and consumer's respectively.

If we have large enough size of the lot then we may use binomial distribution to develop hybrid group acceptance sampling plan. Now the HGASP may be denote as $\left(\mathrm{n}, \mathrm{ct} / \sigma_{0}\right)$. The probability of acceptance is given as:-

$L(p)=\left[\sum_{i=0}^{c}\left(\begin{array}{l}r \\ i\end{array}\right) p^{i}(1-p)^{r-i}\right]^{g}$

Where $\mathrm{p}$ is the probability that an item in a tester fails before the termination time $t_{0}=$ $\mathrm{a} \mu_{0}$.

Now the probability $\mathrm{p}$ for Sushila distribution is given by

$$
\begin{aligned}
& p=F(t)=\frac{1-\mu(\sigma+1)+a \sigma \mu}{\mu(\sigma+1)} e^{-\frac{a \sigma \mu_{0}}{\mu}} \\
& \quad p=F(t)=1-\frac{(\sigma+1)\left(\frac{\mu}{\mu_{0}}\right)+a \sigma \mu_{0}}{(\sigma+1)\left(\frac{\mu}{\mu_{0}}\right)} e^{-} \frac{a \sigma}{\frac{\mu}{\mu_{0}}} \\
& \text { Or } \quad
\end{aligned}
$$

The number of testers $r$ as minimum level can be determine though consumer's risk when the true man life equals to the specified life as $\mu=\mu_{0}$ with the inequality.

$\mathrm{L}\left(\mathrm{p}_{0}\right) \leq \beta$

Where $\mathrm{p}_{0}$ is failure probability with $\mu=\mu_{0}$ and given as follows:

$p=1-\frac{(\sigma+1)+a^{\sigma}}{\sigma-1} e^{-a \sigma}$ 
Srinivasa Rao (2011) proposed a plan for HGASP in detailed. Now, to obtain minimum testers, we have the following table with prefix consumer's risk number of grants, acceptance number and truncation time for sushila distribution.

\section{Operating characteristics}

The operating characteristic function may be regarded as probability of acceptance and denoted by $\mathrm{P}_{\mathrm{a}}$. So, $\mathrm{P}_{\mathrm{a}}$ is a function of the deviation of true man to specified man life. If we have obtained minimum testers then the other parameter is $\mathrm{P}_{\mathrm{a}}$ and it is easy to know the quality of the product. The product is regarded as $\operatorname{good}$ if $\mu>\mu_{0}$.

When $\beta=0.25, g=4, c=2$ and $a=1.0$ the number of testers required is $r=3$. However, if the ratio $\left(\mu / \mu_{0}=2\right)$ then the producer's risk is

$\alpha=1-($ oc value $)=1-0.6465$

$\alpha=0.3535$, while producer's risk $(\alpha)$ is 0.0821 then $\left(\mu / \mu_{0}=4\right)$

The producer is desire to acceptance of the product. So, it should be greater than the given specified level. Now, the minimum ratio $\mu / \mu_{0}$ can be obtained by satisfying the inequality.

$\left[\sum_{0}^{c}\left(\begin{array}{l}r \\ i\end{array}\right) p^{i}(1-p)^{r-i}\right]^{g} \geq 1-\alpha$

$r$ is chosen, given $\mu / \mu_{0}=1$, consumer's risk $(\beta)$ any value from 0.25 to 0.01 .

So, the tables 1 and 2 tell us the behavior of the distribution, minimum sample size of testers and the operating characteristics.

\section{Results and Discussion}

We found many groups and acceptance number for producer's and consumer's specifications in table $1 \& 2$. The design parameters of HGASP are found for various values of consumer's risk i.e. $(\beta=0.25,0.10$, $0.05,0.01)$ when the producer's risk $\alpha=0.05$. It is noticeable that minimum sample size can be obtained through $n=r \times g$. If we increase test termination time multiplier a then number of testers decreases at fixed number of groups.

If the parameters $\mu=\alpha=2$ for certain acceptance numbers in Sushila distribution. The probability of acceptance for the lot of the mean ratio corresponding to the producer's risk also given in table 1 and 2 .

Let us consider that the lifetime of an item follows Sushila Distribution with shape parameter $(\alpha)=2$. The consumer wants that lifetime of an item is greater than 1000 hours on testing time of 700 hours for 6 groups. Now if $\beta=0.10$ and allocate 8 items for 6 groups with acceptance number $\mathrm{c}=4$ put on test 48 items for 700 hours. We will accept the lot if no more than 4 failure occurs before 700 hours in each of 6 groups. We will terminate the procedure as 5th failure occurs before 700th hour. For this sampling plan, $\mathrm{Pa}$ is 0.4165 when the ratio of true mean and specified mean i.e. $\mu / \mu_{0}=2$ or we can say true mean is 2,000 hours. It is clear as above if the true mean life is 2 times of specified life time (i.e. 100 hours) the producer's risk is 0.5835 .

In this paper, we proposed a hybrid group acceptance sampling plan with truncated life test when the item follows Sushila distribution. The hybrid group acceptance sampling plan is applied with good purpose of reducing the cost and inspection time. In this process, we can check multiple items in the group at a time. The minimum number of testers and operating characteristics values represent the behavior of the distribution. This HGASP can be used when a group is considered for life testing. It will provide a future expectations. 
Table.1

\begin{tabular}{|c|c|c|c|c|c|c|}
\hline B & 0.256 & \multicolumn{2}{|c|}{0.10} & \multicolumn{2}{|c|}{0.05} & 0.01 \\
\hline a & 0.7 & 0.8 & 1.0 & 1.2 & 1.5 & 2.0 \\
\hline g & 23 & 45 & & & 89 & 10 \\
\hline c & 01 & 23 & & & 57 & 8 \\
\hline
\end{tabular}

Table.2 Minimum number of testers (r) and acceptance number (c) for the Hybrid group acceptance sampling plans for Sushila distribution

\begin{tabular}{|c|c|c|c|c|c|c|c|c|}
\hline \multirow[t]{2}{*}{$\beta$} & \multirow[t]{2}{*}{$\mathbf{g}$} & \multirow[t]{2}{*}{ c } & \multicolumn{6}{|c|}{$\mathbf{A}$} \\
\hline & & & 0.7 & 0.8 & 1.0 & 1.2 & 1.5 & 2.0 \\
\hline \multirow[t]{9}{*}{0.25} & 2 & 0 & 2 & 2 & 1 & 1 & 1 & 1 \\
\hline & 3 & 1 & 5 & 4 & 2 & 1 & 1 & 1 \\
\hline & 4 & 2 & 7 & 5 & 3 & 2 & 2 & 2 \\
\hline & 5 & 3 & 9 & 6 & 3 & 2 & 2 & 2 \\
\hline & 6 & 4 & 12 & 8 & 5 & 3 & 3 & 2 \\
\hline & 7 & 5 & 15 & 9 & 6 & 4 & 4 & 3 \\
\hline & 8 & 6 & 17 & 11 & 8 & 5 & 4 & 3 \\
\hline & 9 & 7 & 19 & 13 & 9 & 7 & 5 & 4 \\
\hline & 10 & 8 & 21 & 15 & 10 & & & 5 \\
\hline \multirow[t]{9}{*}{0.10} & 2 & 0 & 2 & 2 & 1 & 1 & 1 & 1 \\
\hline & 3 & 1 & 4 & 4 & 2 & 1 & 1 & 1 \\
\hline & 4 & 2 & 6 & 5 & 3 & 2 & 2 & 2 \\
\hline & 5 & 3 & 8 & 6 & 4 & 2 & 2 & 2 \\
\hline & 6 & 4 & 11 & 7 & 5 & 3 & 3 & 2 \\
\hline & 7 & 5 & 13 & 9 & 7 & 5 & 3 & 2 \\
\hline & 8 & 6 & 15 & 12 & 9 & 7 & 4 & 3 \\
\hline & 9 & 7 & 17 & 14 & 10 & 9 & 5 & 4 \\
\hline & 10 & 8 & 19 & 16 & 11 & 11 & 6 & \\
\hline \multirow[t]{9}{*}{0.05} & 2 & 0 & 2 & 2 & 1 & 1 & 1 & 1 \\
\hline & 3 & 1 & 4 & 4 & 2 & 1 & 1 & 1 \\
\hline & 4 & 2 & 6 & 5 & 3 & 2 & 2 & 2 \\
\hline & 5 & 3 & 8 & 6 & 5 & 2 & 2 & 2 \\
\hline & 6 & 4 & 9 & 7 & 7 & 3 & 3 & 2 \\
\hline & 7 & 5 & 11 & 8 & 8 & 3 & 3 & 3 \\
\hline & 8 & 6 & 13 & 9 & 9 & 5 & 4 & 3 \\
\hline & 9 & 7 & 15 & 10 & 10 & 6 & 5 & 4 \\
\hline & 10 & 8 & 17 & 12 & 11 & 7 & 6 & 5 \\
\hline \multirow[t]{9}{*}{0.01} & 2 & 0 & 2 & 2 & 1 & 1 & 1 & 1 \\
\hline & 3 & 1 & 4 & 4 & 2 & 2 & 1 & 1 \\
\hline & 4 & 2 & 6 & 5 & 3 & 3 & 2 & 1 \\
\hline & 5 & 3 & 8 & 6 & 5 & 4 & 3 & 2 \\
\hline & 6 & 4 & 9 & 7 & 7 & 5 & 3 & 3 \\
\hline & 7 & 5 & 10 & 8 & 8 & 7 & 4 & 4 \\
\hline & 8 & 6 & 11 & 9 & 9 & 8 & 5 & 5 \\
\hline & 9 & 7 & 12 & 10 & 10 & 9 & 6 & 6 \\
\hline & 10 & 8 & 13 & 12 & 11 & 10 & 7 & 7 \\
\hline
\end{tabular}


Table.3 Operating characteristics values of the hybrid group sampling plan with $\mathrm{g}=4$ and $\mathrm{c}=2$ for Sushila distribution

\begin{tabular}{|c|c|c|c|c|c|c|c|c|}
\hline $\boldsymbol{\beta}$ & $\mathbf{r}$ & $\mathbf{a}$ & \multicolumn{5}{|c|}{$\boldsymbol{\mu} / \boldsymbol{\mu}_{\mathbf{0}}$} \\
\cline { 3 - 8 } & & & 2 & 4 & 6 & 8 & 10 & 12 \\
\hline $\mathbf{0 . 2 5}$ & 6 & 0.7 & 0.6375 & 0.9093 & 0.9660 & 0.9838 & 0.9911 & 0.9946 \\
& 5 & 0.8 & 0.5993 & 0.8959 & 0.9605 & 0.9811 & 0.9895 & 0.9936 \\
& 4 & 1.0 & 0.6465 & 0.9129 & 0.9676 & 0.9847 & 0.9916 & 0.9949 \\
& 4 & 1.2 & 0.6265 & 0.9028 & 0.9422 & 0.9867 & 0.9927 & 0.9956 \\
& 3 & 1.5 & 0.5058 & 0.8541 & 0.9022 & 0.9505 & 0.9842 & 0.9903 \\
& 3 & 2.0 & 0.3569 & 0.7708 & 0.8540 & 0.9233 & 0.9718 & 0.9824 \\
\hline $\mathbf{0 . 1 0}$ & 8 & 0.7 & 0.4165 & 0.8188 & 0.9223 & 0.9612 & 0.9780 & 0.9864 \\
& 7 & 0.8 & 0.4483 & 0.8296 & 0.9310 & 0.9659 & 0.9808 & 0.9881 \\
& 5 & 1.0 & 0.4501 & 0.8310 & 0.9318 & 0.9564 & 0.9811 & 0.9847 \\
& 4 & 1.2 & 0.3782 & 0.7911 & 0.9129 & 0.9130 & 0.9753 & 0.9676 \\
& 4 & 1.5 & 0.3569 & 0.7708 & 0.9022 & 0.9505 & 0.9489 & 0.9824 \\
& 3 & 2.0 & 0.2433 & 0.6812 & 0.8540 & 0.9233 & 0.9553 & 0.9718 \\
\hline $\mathbf{0 . 0 5}$ & 9 & 0.7 & 0.3263 & 0.7561 & 0.8946 & 0.9461 & 0.9690 & 0.9806 \\
& 7 & 0.8 & 0.3230 & 0.7552 & 0.8944 & 0.9461 & 0.9659 & 0.9806 \\
& 6 & 1.0 & 0.2952 & 0.7361 & 0.8848 & 0.9409 & 0.9753 & 0.9786 \\
& 5 & 1.2 & 0.3124 & 0.7911 & 0.9129 & 0.9564 & 0.9489 & 0.9847 \\
& 4 & 1.5 & 0.3782 & 0.6467 & 0.9022 & 0.9505 & 0.9718 & 0.9676 \\
& 4 & 2.0 & 0.3569 & 0.6812 & 0.8540 & 0.9233 & 0.9553 & 0.9718 \\
\hline $\mathbf{0 . 0 1}$ & 10 & 0.7 & 0.1901 & 0.6401 & 0.8301 & 0.9090 & 0.9461 & 0.9656 \\
& 9 & 0.8 & 0.2259 & 0.6771 & 0.8522 & 0.9221 & 0.9544 & 0.9664 \\
& 7 & 1.0 & 0.1852 & 0.6371 & 0.8293 & 0.9088 & 0.9471 & 0.9676 \\
& 6 & 1.2 & 0.1861 & 0.6390 & 0.8311 & 0.9102 & 0.9489 & 0.9435 \\
& 5 & 1.5 & 0.1752 & 0.6467 & 0.8357 & 0.9130 & 0.9129 & 0.9329 \\
& 4 & 2.0 & 0.0928 & 0.3782 & 0.6466 & 0.7911 & 0.8688 & 0.9129 \\
\hline
\end{tabular}

This process may be represent through a flow chart as follows:

Select the number of testers (r)

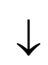

Assign $\mathrm{r}$ to each group g i.e. $\mathrm{n}=\mathrm{gr}$ and put into test for to

Calculate $\mathrm{d}$ as defective for each group

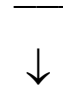

Accept the lot if $\leq \mathrm{c}$ in each group
Reject the lot if $\mathrm{d}>\mathrm{c}$ in any group 
However, this approach may be applied for other life time distributions with this hybrid group acceptance sampling plan.

\section{Acknowledgements}

The author is thankful to the referees and the editors for their valuable comments.

\section{References}

1. Epstein, B. (1954). Truncated life tests in the exponential case. Annals of Mathematical statistics, 25, 555 - 564.

2. Goode, H.P. and Kao, J.H.K (1961). Sampling plans based on the Weibull distribution. Proceeding of the $7^{\text {th }}$ National Symposium on Reliability and Quality Control, 24 - 40, Philadelphia.

3. Gupta, S.S. (1962). Life test sampling plans for normal and lognormal distribution, Technometrics, 4(2), 151 175.

4. Gupta, S.S. and Groll, P.A. (1961). Gamma distribution in acceptance sampling based on life tests. J. Amer. Statist. Assoc., 56, $942-970$.

5. Aslam, M. and C.H. Jun (2009).A group acceptance Sampling plan for truncated life testing having Weibull distribution. J. Appl. Stat, 36 (9), 1021 - 1027.

6. Muhammad, ADebasis, $\mathrm{K}$ and Munit, A (2010).Time truncated acceptance sampling plans for generalized exponential distribution. Pak. J. Commer. Soc. Sci. 1, 1-20.

7. Srinivasa Rao, G. (zolo). A group acceptance sampling plans for truncated life tests for Marshall - Olkin extended Lomax distribution. Electronic journal of applied statistical analysis, 3 (1), $18-27$.

8. Srinivasea Rao, G. (2011). A hybrid group acceptance sampling plans for lifetime based on $\log$ - logistic distribution. Journal of reliability and statistical studies, 4(1), $31-40$.

9. Al Omani, A.I. (2018).Acceptance sampling plans based on truncated life test for Sushila distribution. J. Math. Fund. Sci. 50(1), $72-73$.

10. Subba Rao, R. Naga Durgamamba, A., Kantam, R.R.L. (2014). Hybrid group acceptance sampling plan based on size biased Lomax model. Mathematics and Statistics, 2 (3), 137 - 141.

11. Raj Gopal, S., Vijayadevi, K. (2018).A hybrid group acceptance sampling plan for lifetimes based on transmuted rayleigh distribution. International Journal of Scientific Research in Mathematical and Statistical Science, 5(4), 77 - 94.

12. Shanker, R., Sharma, S., Shanker, U. and Shanker, R. (2013). Sushila Distribution and Its Application to Waiting Times Data. International Journal of Business Management, 3(2), 1-11.

\section{How to cite this article:}

Sandeep Kumar. 2019. Hybrid Group Acceptance Sampling Plan on Truncated Life Tests based on Sushila Distribution. Int.J.Curr.Microbiol.App.Sci. 8(08): 418-424. doi: https://doi.org/10.20546/ijcmas.2019.808.047 